\title{
Lyapunov-type inequalities and disconjugacy for some nonlinear difference system
}

Qi-Ming Zhang ${ }^{1 *}$, Xiaofei $\mathrm{He}^{2}$ and XH Tang ${ }^{2}$

${ }^{*}$ Correspondence:
zhqm20082008@sina.com
${ }^{1}$ College of Science, Hunan
University of Technology, Zhuzhou,
Hunan 412007, P.R. China
Full list of author information is
available at the end of the article

available at the end of the article

\begin{abstract}
We establish several new Lyapunov-type inequalities for some nonlinear difference system when the end-points are not necessarily usual zeros, but rather generalized zeros. Our results generalize in some sense the known ones. As an application, we develop disconjugacy criteria by making use of the obtained inequalities.

MSC: 34D20; 39A99

Keywords: difference system; nonlinearity; Lyapunov-type inequality; generalized zero; disconjugacy
\end{abstract}

\section{Introduction}

Consider the following nonlinear difference system:

$$
\left\{\begin{array}{l}
\triangle x(n)=\alpha(n) x(n+1)+\beta(n)|y(n)|^{\mu-2} y(n), \\
\triangle y(n)=-\gamma(n)|x(n+1)|^{\nu-2} x(n+1)-\alpha(n) y(n),
\end{array}\right.
$$

where $\mu, v>1$ and $\frac{1}{\mu}+\frac{1}{v}=1, \alpha(n), \beta(n)$ and $\gamma(n)$ are real-valued functions defined on $\mathbb{Z}$, $\triangle$ denotes the forward difference operator defined by $\Delta x(n)=x(n+1)-x(n)$. Throughout this paper, we always assume that

$$
\beta(n) \geq 0, \quad \forall n \in \mathbb{Z}
$$

When $\mu=v=2$, system (1.1) reduces to a discrete linear Hamiltonian system

$$
\triangle x(n)=\alpha(n) x(n+1)+\beta(n) y(n), \quad \triangle y(n)=-\gamma(n) x(n+1)-\alpha(n) y(n) .
$$

Similar to [1], we first give the following two definitions.

Definition 1.1 [1] A function $f: \mathbb{Z} \rightarrow \mathbb{R}$ is said to have a generalized zero at $n_{0} \in \mathbb{Z}$ provided either $f\left(n_{0}\right)=0$ or $f\left(n_{0}\right) f\left(n_{0}+1\right)<0$.

Definition 1.2 [1] Let $a, b \in \mathbb{Z}$ with $a \leq b-2$. A function $f: \mathbb{Z}[a, b] \rightarrow \mathbb{R}$ is said to be disconjugate if it has at most a generalized zero on $\mathbb{Z}[a, b]$; otherwise, it is conjugate on $\mathbb{Z}[a, b]$.

@ 2013 Zhang et al.; licensee Springer. This is an Open Access article distributed under the terms of the Creative Commons Attribution License (http://creativecommons.org/licenses/by/2.0), which permits unrestricted use, distribution, and reproduction in any medium, provided the original work is properly cited. 
In addition, the definition that system (1.1) is relatively disconjugate here is the same as Definition 4 in [1].

For system (1.1), there are some generalizations and extensions related to Lyapunovtype inequalities; and for recent work in the literature on discrete and continuous cases as well as its special case, i.e., system (1.3), we refer to [1-6] and the references therein. Based on [3] and [5], we further discuss system (1.1) in this paper and establish some new Lyapunov-type inequalities. In [1], the authors have gained many interesting results about Lyapunov-type inequalities and their applications for system (1.3) including developing several disconjugacy criteria by adopting some techniques. As an application, we also develop disconjugacy criteria in the last section. Moreover, we make a comparison with some existing ones.

Now, we state several relative results in [1] and [3].

Theorem 1.3 [3] Suppose that (1.2) holds and

$$
1-\alpha(n)>0, \quad \forall n \in \mathbb{Z}
$$

Let $n \in \mathbb{Z}[a, b]$ with $a \leq b-1$. Assume (1.1) has a real solution $(x(n), y(n))$ satisfying

$$
\begin{aligned}
& x(a)=0 \quad \text { or } \quad x(a) x(a+1)<0 ; \\
& x(b)=0 \quad \text { or } \quad x(b) x(b+1)<0 ; \quad \max _{a+1 \leq n \leq b}|x(n)|>0 .
\end{aligned}
$$

Then one has the following Lyapunov-type inequality:

$$
\sum_{n=a}^{b-1}|\alpha(n)|+\left(\sum_{n=a}^{b} \beta(n)\right)^{1 / \mu}\left(\sum_{n=a}^{b-1} \gamma^{+}(n)\right)^{1 / \nu} \geq 2,
$$

where, and in what follows, $\mathbb{Z}[a, b]=\{a, a+1, \ldots, b+1, b\}$ for any $a, b \in \mathbb{Z}$ with $a<b$ and $\gamma^{+}(n)=\max \{\gamma(n), 0\}$.

Theorem 1.4 [3] Suppose that (1.2) and (1.4) hold and let $n \in \mathbb{Z}[a, b]$ with $a \leq b-2$. Assume (1.1) has a real solution $(x(n), y(n))$ such that $x(a)=0$ or $x(a) x(a+1)<0$ and $x(b)=0$ and $x(n)$ is not identically zero on $[a, b]$. Then one has the following Lyapunov-type inequality:

$$
\sum_{n=a}^{b-2}|\alpha(n)|+\left(\sum_{n=a}^{b-1} \beta(n)\right)^{1 / \mu}\left(\sum_{n=a}^{b-2} \gamma^{+}(n)\right)^{1 / \nu} \geq 2
$$

Theorem 1.5 [1] Suppose that (1.4) holds and

$$
\beta(n)>0, \quad \forall n \in \mathbb{Z}[a, b] .
$$

If

$$
\left(\sum_{n=a}^{b} \beta(n) e_{-\alpha}^{2}(n, \tau)\right)\left(\sum_{n=a}^{b-1} \gamma^{+}(n)\right) \leq 1
$$

holds for all $\tau \in \mathbb{Z}[a+1, b]$, then system (1.3) is relatively disconjugate on $\mathbb{Z}[a, b+1]$. 
Regarding the discrete exponential function $e_{-\alpha}(n, \tau)$ of $(1.9)$, we refer the reader to [1].

\section{Lyapunov-type inequalities}

In this section, we establish some new Lyapunov-type inequalities.

Denote

$$
\begin{aligned}
& \zeta(n):=\left\{\sum_{\tau=a}^{n} \beta(\tau) \prod_{s=\tau}^{n}[1-\alpha(s)]^{-\mu}\right\}^{\frac{\nu}{\mu}}, \\
& \eta(n):=\left\{\sum_{\tau=n+1}^{b} \beta(\tau) \prod_{s=n+1}^{\tau-1}[1-\alpha(s)]^{\mu}\right\}^{\frac{\nu}{\mu}},
\end{aligned}
$$

and for $\lambda \in[0,1)$, denote

$$
\begin{aligned}
& \zeta_{\lambda}(n):=\left\{(1-\lambda) \beta(a) \prod_{s=a}^{n}[1-\alpha(s)]^{-\mu}+\sum_{\tau=a+1}^{n} \beta(\tau) \prod_{s=\tau}^{n}[1-\alpha(s)]^{-\mu}\right\}^{\frac{v}{\mu}}, \\
& \eta_{\lambda}(n):=\left\{\lambda \beta(a) \prod_{s=n+1}^{b-1}[1-\alpha(s)]^{\mu}+\sum_{\tau=n+1}^{b-1} \beta(\tau) \prod_{s=n+1}^{\tau-1}[1-\alpha(s)]^{\mu}\right\}^{\frac{\nu}{\mu}} .
\end{aligned}
$$

Theorem 2.1 Suppose that (1.2) and (1.4) hold, and let $n \in \mathbb{Z}[a, b]$ with $a \leq b-1$. Assume (1.1) has a real solution $(x(n), y(n))$ such that (1.5) holds. Then one has the following inequality:

$$
\sum_{n=a}^{b-1} \frac{\zeta(n) \eta(n)}{\zeta(n)+\eta(n)} \gamma^{+}(n) \geq 1 .
$$

Proof It follows from (1.5) that there exist $\xi_{1}, \xi_{2} \in[0,1)$ such that

$$
\left(1-\xi_{1}\right) x(a)+\xi_{1} x(a+1)=0
$$

and

$$
\left(1-\xi_{2}\right) x(b)+\xi_{2} x(b+1)=0 .
$$

Multiplying the first equation of (1.1) by $y(n)$ and the second one by $x(n+1)$, and then adding, we get

$$
\triangle[x(n) y(n)]=\beta(n)|y(n)|^{\mu}-\gamma(n)|x(n+1)|^{v} .
$$

Summing equation (2.8) from $a$ to $b-1$, we can obtain

$$
x(b) y(b)-x(a) y(a)=\sum_{n=a}^{b-1} \beta(n)|y(n)|^{\mu}-\sum_{n=a}^{b-1} \gamma(n)|x(n+1)|^{\nu} .
$$

From the first equation of (1.1), we have

$$
[1-\alpha(n)] x(n+1)=x(n)+\beta(n)|y(n)|^{\mu-2} y(n) .
$$


Combining (2.10) with (2.6), we have

$$
x(a)=-\frac{\xi_{1} \beta(a)}{1-\left(1-\xi_{1}\right) \alpha(a)}|y(a)|^{\mu-2} y(a) .
$$

Similarly, it follows from (2.10) and (2.7) that

$$
x(b)=-\frac{\xi_{2} \beta(b)}{1-\left(1-\xi_{2}\right) \alpha(b)}|y(b)|^{\mu-2} y(b) .
$$

Substituting (2.11) and (2.12) into (2.9), we have

$$
\begin{aligned}
& \sum_{n=a}^{b-1} \beta(n)|y(n)|^{\mu}-\sum_{n=a}^{b-1} \gamma(n)|x(n+1)|^{\nu} \\
& \quad=-\frac{\xi_{2} \beta(b)}{1-\left(1-\xi_{2}\right) \alpha(b)}|y(b)|^{\mu}+\frac{\xi_{1} \beta(a)}{1-\left(1-\xi_{1}\right) \alpha(a)}|y(a)|^{\mu}
\end{aligned}
$$

which implies that

$$
\begin{aligned}
& \frac{\left(1-\xi_{1}\right)[1-\alpha(a)]}{1-\left(1-\xi_{1}\right) \alpha(a)} \beta(a)|y(a)|^{\mu}+\sum_{n=a+1}^{b-1} \beta(n)|y(n)|^{\mu}+\frac{\xi_{2} \beta(b)}{1-\left(1-\xi_{2}\right) \alpha(b)}|y(b)|^{\mu} \\
& \quad=\sum_{n=a}^{b-1} \gamma(n)|x(n+1)|^{v} .
\end{aligned}
$$

Denote that

$$
\tilde{\beta}(a)=\frac{\left(1-\xi_{1}\right)[1-\alpha(a)]}{1-\left(1-\xi_{1}\right) \alpha(a)} \beta(a), \quad \tilde{\beta}(b)=\frac{\xi_{2}}{1-\left(1-\xi_{2}\right) \alpha(b)} \beta(b)
$$

and

$$
\tilde{\beta}(n)=\beta(n), \quad n \in \mathbb{Z}[a+1, b-1] .
$$

Then we can rewrite (2.13) as

$$
\sum_{n=a}^{b} \tilde{\beta}(n)|y(n)|^{\mu}=\sum_{n=a}^{b-1} \gamma(n)|x(n+1)|^{\nu}
$$

From (2.10), (2.11), (2.14) and (2.15), we obtain

$$
\begin{aligned}
x(n+1)= & x(a) \prod_{s=a}^{n}[1-\alpha(s)]^{-1}+\sum_{\tau=a}^{n} \beta(\tau)|y(\tau)|^{\mu-2} y(\tau) \prod_{s=\tau}^{n}[1-\alpha(s)]^{-1} \\
= & -\frac{\xi_{1} \beta(a)}{1-\left(1-\xi_{1}\right) \alpha(a)}|y(a)|^{\mu-2} y(a) \prod_{s=a}^{n}[1-\alpha(s)]^{-1} \\
& +\sum_{\tau=a}^{n} \beta(\tau)|y(\tau)|^{\mu-2} y(\tau) \prod_{s=\tau}^{n}[1-\alpha(s)]^{-1} \\
= & \sum_{\tau=a}^{n} \tilde{\beta}(\tau)|y(\tau)|^{\mu-2} y(\tau) \prod_{s=\tau}^{n}[1-\alpha(s)]^{-1}, \quad n \in \mathbb{Z}[a, b-1] .
\end{aligned}
$$


Similarly, from (2.10), (2.12), (2.14) and (2.15), we have

$$
\begin{aligned}
x(n+1)= & x(b) \prod_{s=n+1}^{b-1}[1-\alpha(s)]-\sum_{\tau=n+1}^{b-1} \beta(\tau)|y(\tau)|^{\mu-2} y(\tau) \prod_{s=n+1}^{\tau-1}[1-\alpha(s)] \\
= & -\frac{\xi_{2} \beta(b)}{1-\left(1-\xi_{2}\right) \alpha(b)}|y(b)|^{\mu-2} y(b) \prod_{s=n+1}^{b-1}[1-\alpha(s)] \\
& -\sum_{\tau=n+1}^{b-1} \beta(\tau)|y(\tau)|^{\mu-2} y(\tau) \prod_{s=n+1}^{\tau-1}[1-\alpha(s)] \\
= & -\sum_{\tau=n+1}^{b} \tilde{\beta}(\tau)|y(\tau)|^{\mu-2} y(\tau) \prod_{s=n+1}^{\tau-1}[1-\alpha(s)], \quad n \in \mathbb{Z}[a, b-1] .
\end{aligned}
$$

Since

$$
0 \leq \tilde{\beta}(n) \leq \beta(n), \quad n \in \mathbb{Z}[a, b]
$$

it follows from (2.1) and (2.17) and the Hölder inequality that

$$
\begin{aligned}
|x(n+1)|^{\nu} & \leq\left\{\sum_{\tau=a}^{n} \tilde{\beta}(\tau)|y(\tau)|^{\mu-1} \prod_{s=\tau}^{n}[1-\alpha(s)]^{-1}\right\}^{\nu} \\
& \leq\left(\sum_{\tau=a}^{n} \tilde{\beta}(\tau) \prod_{s=\tau}^{n}[1-\alpha(s)]^{-\mu}\right)^{\frac{\nu}{\mu}} \sum_{\tau=a}^{n} \tilde{\beta}(\tau)|y(\tau)|^{\mu} \\
& \leq\left(\sum_{\tau=a}^{n} \beta(\tau) \prod_{s=\tau}^{n}[1-\alpha(s)]^{-\mu}\right)^{\frac{v}{\mu}} \sum_{\tau=a}^{n} \tilde{\beta}(\tau)|y(\tau)|^{\mu} \\
& =\zeta(n) \sum_{\tau=a}^{n} \tilde{\beta}(\tau)|y(\tau)|^{\mu}, \quad n \in \mathbb{Z}[a, b-1] .
\end{aligned}
$$

Similarly, it follows from (2.2), (2.18), (2.19) and the Hölder inequality that

$$
\begin{aligned}
|x(n+1)|^{\nu} & \leq\left\{\sum_{\tau=n+1}^{b} \tilde{\beta}(\tau)|y(\tau)|^{\mu-1} \prod_{s=n+1}^{\tau-1}[1-\alpha(s)]\right\}^{v} \\
& \leq\left(\sum_{\tau=n+1}^{b} \tilde{\beta}(\tau) \prod_{s=n+1}^{\tau-1}[1-\alpha(s)]^{\mu}\right)^{\frac{\nu}{\mu}} \sum_{\tau=n+1}^{b} \tilde{\beta}(\tau)|y(\tau)|^{\mu} \\
& \leq\left(\sum_{\tau=n+1}^{b} \beta(\tau) \prod_{s=n+1}[1-\alpha(s)]^{\mu}\right)^{\frac{v}{\mu}} \sum_{\tau=n+1}^{b} \tilde{\beta}(\tau)|y(\tau)|^{\mu} \\
& =\eta(n) \sum_{\tau=n+1}^{b} \tilde{\beta}(\tau)|y(\tau)|^{\mu}, \quad n \in \mathbb{Z}[a, b-1] .
\end{aligned}
$$

From (2.20) and (2.21), we obtain

$$
|x(n+1)|^{\nu} \leq \frac{\zeta(n) \eta(n)}{\zeta(n)+\eta(n)} \sum_{\tau=a}^{b} \tilde{\beta}(\tau)|y(\tau)|^{\mu}, \quad n \in \mathbb{Z}[a, b-1]
$$


Combining (2.22) with (2.16), we have

$$
\begin{aligned}
\sum_{n=a}^{b-1} \gamma^{+}(n)|x(n+1)|^{\nu} & \leq \sum_{n=a}^{b-1} \frac{\zeta(n) \eta(n)}{\zeta(n)+\eta(n)} \gamma^{+}(n) \sum_{n=a}^{b} \tilde{\beta}(n)|y(\tau)|^{\mu} \\
& =\sum_{n=a}^{b-1} \frac{\zeta(n) \eta(n)}{\zeta(n)+\eta(n)} \gamma^{+}(n) \sum_{n=a}^{b-1} \gamma(n)|x(n+1)|^{\nu} \\
& \leq \sum_{n=a}^{b-1} \frac{\zeta(n) \eta(n)}{\zeta(n)+\eta(n)} \gamma^{+}(n) \sum_{n=a}^{b-1} \gamma^{+}(n)|x(n+1)|^{\nu} .
\end{aligned}
$$

We claim that

$$
\sum_{n=a}^{b-1} \gamma^{+}(n)|x(n+1)|^{v}>0
$$

If (2.24) is not true, then

$$
\sum_{n=a}^{b-1} \gamma^{+}(n)|x(n+1)|^{\nu}=0
$$

From (2.16) and (2.25), we have

$$
0 \leq \sum_{n=a}^{b} \tilde{\beta}(n)|y(n)|^{\mu}=\sum_{n=a}^{b-1} \gamma(n)|x(n+1)|^{\nu} \leq \sum_{n=a}^{b-1} \gamma^{+}(n)|x(n+1)|^{\nu}=0 .
$$

It follows that

$$
\tilde{\beta}(n)|y(n)|^{\mu}=0, \quad n \in \mathbb{Z}[a, b] .
$$

Combining (2.20) with (2.27), we obtain that $x(a+1)=x(a+2)=\cdots=x(b)=0$, which together with (2.6) implies that $x(a)=0$. This contradicts (1.5). Therefore, (2.24) holds. Hence, it follows from (2.23) and (2.24) that (2.5) holds.

In the case $x(b)=0$, i.e., $\xi_{2}=0$, and so $\tilde{\beta}(b)=0$, we have the following equation:

$$
\sum_{n=a}^{b-1} \tilde{\beta}(n)|y(n)|^{\mu}=\sum_{n=a}^{b-2} \gamma(n)|x(n+1)|^{v}
$$

and inequality

$$
\begin{aligned}
|x(n+1)|^{\nu} & \leq\left\{\sum_{\tau=n+1}^{b-1} \tilde{\beta}(\tau)|y(\tau)|^{\mu-1} \prod_{s=n+1}^{\tau-1}[1-\alpha(s)]\right\}^{\nu} \\
& \leq\left(\sum_{\tau=n+1}^{b-1} \tilde{\beta}(\tau) \prod_{s=n+1}^{\tau-1}[1-\alpha(s)]^{\mu}\right)^{\frac{\nu}{\mu}} \sum_{\tau=n+1}^{b-1} \tilde{\beta}(\tau)|y(\tau)|^{\mu}
\end{aligned}
$$




$$
\begin{aligned}
& \leq\left(\sum_{\tau=n+1}^{b-1} \beta(\tau) \prod_{s=n+1}^{\tau-1}[1-\alpha(s)]^{\mu}\right)^{\frac{\nu}{\mu}} \sum_{\tau=n+1}^{b-1} \tilde{\beta}(\tau)|y(\tau)|^{\mu} \\
& =\eta_{0}(n) \sum_{\tau=n+1}^{b-1} \tilde{\beta}(\tau)|y(\tau)|^{\mu}, \quad n \in \mathbb{Z}[a, b-1]
\end{aligned}
$$

instead of (2.16) and (2.21), respectively. Similar to the proof of Theorem 2.1, we have the following theorem.

Theorem 2.2 Suppose that (1.2) and (1.4) hold, and let $n \in \mathbb{Z}[a, b]$ with $a \leq b-2$. Assume (1.1) has a real solution $(x(n), y(n))$ such that $x(a)=0$ or $x(a) x(a+1)<0$ and $x(b)=0$ and $x(n)$ is not identically zero on $\mathbb{Z}[a, b]$. Then one has the following inequality:

$$
\sum_{n=a}^{b-2} \frac{\zeta(n) \eta_{0}(n)}{\zeta(n)+\eta_{0}(n)} \gamma^{+}(n) \geq 1
$$

where $\zeta(n)$ and $\eta_{0}(n)$ are defined by (2.1) and (2.4), respectively.

Corollary 2.3 Suppose that (1.2) and (1.4) hold, and let $n \in \mathbb{Z}[a, b]$ with $a \leq b-1$. Assume (1.1) has a real solution $(x(n), y(n))$ such that (1.5) holds. Then one has the following inequality:

$$
\sum_{n=a}^{b-1} \gamma^{+}(n)\left[\sum_{\tau=a}^{n} \beta(\tau) \sum_{\tau=n+1}^{b} \beta(\tau)\right]^{\frac{\nu}{2 \mu}} \geq 2\left\{\prod_{n=a}^{b-1} \Theta[\alpha(n)]\right\}^{\frac{\nu}{2}}
$$

where, and in the sequel,

$$
\Theta[\alpha(n)]=\min \left\{1-\alpha^{+}(n),\left[1+\alpha^{-}(n)\right]^{-1}\right\}
$$

and

$$
\alpha^{+}(n)=\max \{\alpha(n), 0\}, \quad \alpha^{-}(n)=\max \{-\alpha(n), 0\} .
$$

Proof Since

$$
\zeta(n)+\eta(n) \geq 2[\zeta(n) \eta(n)]^{\frac{1}{2}}
$$

it follows that

$$
\begin{aligned}
1 & \leq \sum_{n=a}^{b-1} \frac{\zeta(n) \eta(n)}{\zeta(n)+\eta(n)} \gamma^{+}(n) \\
& \leq \frac{1}{2} \sum_{n=a}^{b-1}[\zeta(n) \eta(n)]^{\frac{1}{2}} \gamma^{+}(n) \\
& =\frac{1}{2} \sum_{n=a}^{b-1} \gamma^{+}(n)\left\{\sum_{\tau=a}^{n} \beta(\tau) \prod_{s=\tau}^{n}[1-\alpha(s)]^{-\mu} \sum_{\tau=n+1}^{b} \beta(\tau) \prod_{s=n+1}^{\tau-1}[1-\alpha(s)]^{\mu}\right\}^{\frac{\nu}{2 \mu}}
\end{aligned}
$$




$$
\begin{aligned}
& \leq \frac{1}{2} \sum_{n=a}^{b-1} \gamma^{+}(n)\left\{\sum_{\tau=a}^{n} \beta(\tau) \prod_{s=\tau}^{n}\left[1-\alpha^{+}(s)\right]^{-\mu} \sum_{\tau=n+1}^{b} \beta(\tau) \prod_{s=n+1}^{\tau-1}\left[1+\alpha^{-}(s)\right]^{\mu}\right\}^{\frac{v}{2 \mu}} \\
& \leq \frac{1}{2} \sum_{n=a}^{b-1} \gamma^{+}(n)\left[\sum_{\tau=a}^{n} \beta(\tau) \sum_{\tau=n+1}^{b} \beta(\tau)\right]^{\frac{v}{2 \mu}} \prod_{s=a}^{n}\left[1-\alpha^{+}(s)\right]^{-\frac{v}{2}} \prod_{s=n+1}^{b-1}\left[1+\alpha^{-}(s)\right]^{\frac{v}{2}} \\
& \leq \frac{1}{2} \sum_{n=a}^{b-1} \gamma^{+}(n)\left[\sum_{\tau=a}^{n} \beta(\tau) \sum_{\tau=n+1}^{b} \beta(\tau)\right]^{\frac{v}{2 \mu}}\left\{\prod_{s=a}^{b-1} \Theta[\alpha(s)]\right\}^{-\frac{v}{2}},
\end{aligned}
$$

which implies (2.31) holds.

Since

$$
\left[\sum_{\tau=a}^{n} \beta(\tau) \sum_{\tau=n+1}^{b} \beta(\tau)\right]^{\frac{1}{2}} \leq \frac{1}{2} \sum_{n=a}^{b} \beta(n)
$$

we have the following result.

Corollary 2.4 Suppose that (1.2) and (1.4) hold, and let $n \in \mathbb{Z}[a, b]$ with $a \leq b-1$. Assume (1.1) has a real solution $(x(n), y(n))$ such that (1.5) holds. Then one has the following Lyapunov-type inequality:

$$
\left(\sum_{n=a}^{b} \beta(n)\right)^{\frac{1}{\mu}}\left(\sum_{n=a}^{b-1} \gamma^{+}(n)\right)^{\frac{1}{v}} \geq 2\left\{\prod_{n=a}^{b-1} \Theta[\alpha(n)]\right\}^{\frac{1}{2}} .
$$

In a fashion similar to the proofs of Corollaries 2.3 and 2.4, we can prove the following corollaries by using Theorem 2.2 instead of Theorem 2.1.

Corollary 2.5 Suppose that (1.2) and (1.4) hold, and let $n \in \mathbb{Z}[a, b]$ with $a \leq b-2$. Assume (1.1) has a real solution $(x(n), y(n))$ such that $x(a)=0$ or $x(a) x(a+1)<0$ and $x(b)=0$ and $x(n)$ is not identically zero on $\mathbb{Z}[a, b]$. Then one has the following inequality:

$$
\sum_{n=a}^{b-2} \gamma^{+}(n)\left[\sum_{\tau=a}^{n} \beta(\tau) \sum_{\tau=n+1}^{b-1} \beta(\tau)\right]^{\frac{v}{2 \mu}} \geq 2\left\{\prod_{n=a}^{b-2} \Theta[\alpha(n)]\right\}^{\frac{\nu}{2}} .
$$

Corollary 2.6 Suppose that (1.2) and (1.4) hold, and let $n \in \mathbb{Z}[a, b]$ with $a \leq b-2$. Assume (1.1) has a real solution $(x(n), y(n))$ such that $x(a)=0$ or $x(a) x(a+1)<0$ and $x(b)=0$ and $x(n)$ is not identically zero on $\mathbb{Z}[a, b]$. Then one has the following Lyapunov-type inequality:

$$
\left(\sum_{n=a}^{b-1} \beta(n)\right)^{\frac{1}{\mu}}\left(\sum_{n=a}^{b-2} \gamma^{+}(n)\right)^{\frac{1}{v}} \geq 2\left\{\prod_{n=a}^{b-2} \Theta[\alpha(n)]\right\}^{\frac{1}{2}} .
$$

Remark 2.7 While the coefficient $\alpha(n) \leq 0$ and $\mu=v=2$ in system (1.1), inequality (1.6) of Theorem 1.3 can be derived from inequality (2.33). Similarly, for inequality (1.7) of Theorem 1.4 and inequality (2.35), this result also holds. 
On the one hand, when $\alpha(n) \leq 0$, according to the definition of $\Theta[\alpha(n)]$, we have

$$
\Theta[\alpha(n)]=\min \left\{1-\alpha^{+}(n),\left[1+\alpha^{-}(n)\right]^{-1}\right\}=[1+|\alpha(n)|]^{-1} .
$$

On the other hand, for any $u \geq 0$, we have

$$
\ln (1+u) \leq u
$$

and

$$
e^{-u} \geq 1-u
$$

Using above inequalities (2.36), (2.37) and (2.38), we obtain

$$
\begin{aligned}
2\left\{\prod_{n=a}^{b-1} \Theta[\alpha(n)]\right\}^{\frac{1}{2}} & =2\left\{\prod_{n=a}^{b-1}[1+|\alpha(n)|]^{-1}\right\}^{\frac{1}{2}} \\
& =2 \exp \left\{-\ln \left[\prod_{n=a}^{b-1}(1+|\alpha(n)|)^{\frac{1}{2}}\right]\right\} \\
& \geq 2 \exp \left\{-\frac{1}{2} \sum_{n=a}^{b-1}|\alpha(n)|\right\} \\
& \geq 2\left[1-\frac{1}{2} \sum_{n=a}^{b-1}|\alpha(n)|\right]=2-\sum_{n=a}^{b-1}|\alpha(n)| .
\end{aligned}
$$

Then Remark 2.7 holds immediately.

\section{Disconjugacy criteria}

Let $a, b \in \mathbb{Z}$ with $a \leq b-2$. For system (1.1), we develop the following disconjugacy criterion on $\mathbb{Z}[a, b]$ in this section.

Theorem 3.1 Assume that (1.2) and (1.4) hold. If

$$
\left(\sum_{n=a}^{b} \beta(n)\right)^{\frac{1}{\mu}}\left(\sum_{n=a}^{b-1} \gamma^{+}(n)\right)^{\frac{1}{v}}<2\left\{\prod_{n=a}^{b-1} \Theta[\alpha(n)]\right\}^{\frac{1}{2}}
$$

holds, then system (1.1) is relatively disconjugate on $\mathbb{Z}[a, b+1]$.

Proof Suppose that system (1.1) is not relatively disconjugate on $\mathbb{Z}[a, b+1]$. Then there is a real solution $(x, y)$ with $x$ nontrivial and containing two generalized zeros at least. Without loss of generality, we assume that $x(a)=0$ or $x(a) x(a+1)<0$ and the next generalized zero at $c \in \mathbb{Z}[a+2, b]$, i.e., $x(c)=0$ or $x(c) x(c+1)<0$. Hence, applying Corollary 2.4 , we have

$$
\left(\sum_{n=a}^{c} \beta(n)\right)^{\frac{1}{\mu}}\left(\sum_{n=a}^{c-1} \gamma^{+}(n)\right)^{\frac{1}{v}} \geq 2\left\{\prod_{n=a}^{c-1} \Theta[\alpha(n)]\right\}^{\frac{1}{2}},
$$

which clearly contradicts (3.1). 
When $\mu=v=2$ in system (1.1), then for system (1.3), the corresponding disconjugate condition (3.1) reduces to

$$
\sum_{n=a}^{b} \beta(n) \sum_{n=a}^{b-1} \gamma^{+}(n)<4 \prod_{n=a}^{b-1} \Theta[\alpha(n)] .
$$

While $\alpha(n)=0$, system (1.3) reduces to

$$
\triangle x(n)=\beta(n) y(n), \quad \Delta y(n)=-\gamma(n) x(n+1) .
$$

Then disconjugate conditions (1.9) and (3.3) reduce to

$$
\sum_{n=a}^{b} \beta(n) \sum_{n=a}^{b-1} \gamma^{+}(n) \leq 1
$$

and

$$
\sum_{n=a}^{b} \beta(n) \sum_{n=a}^{b-1} \gamma^{+}(n)<4
$$

respectively.

Remark 3.2 For system (3.4), it is obvious that disconjugate condition (3.5) implies that disconjugate condition (3.6) holds, but (3.5) cannot be derived from (3.6). Moreover, condition (1.2) is weaker than condition (1.8).

It is well-known that the second-order difference equation

$$
\triangle^{2} x(n)+q(n) x(n+1)=0
$$

is just a special case of system (3.4) when $\beta(n)=1, \gamma(n)=q(n)$. And so, we have the following corollary.

Corollary 3.3 Assume that (1.2) and (1.4) hold. If inequality (3.6) holds, then equation (3.7) is relatively disconjugate on $\mathbb{Z}[a, b+1]$.

Competing interests

The authors declare that they have no competing interests.

Authors' contributions

All authors carried out the proof and conceived of the study. All authors read and approved the final manuscript.

\section{Author details}

${ }^{1}$ College of Science, Hunan University of Technology, Zhuzhou, Hunan 412007, P.R. China. ${ }^{2}$ School of Mathematical

Sciences and Computing Technology, Central South University, Changsha, Hunan 410083, P.R. China.

\section{Acknowledgements}

The authors thank the referees for valuable comments and suggestions which improved the presentation of this manuscript. This study was partially supported by the National Natural Science Foundation of China (No: 11201138) and the Scientific Research Fund of Hunan Provincial Education Department (No: 12B034). 
References

1. Mert, R, Zafer, A: On stability and disconjugacy criteria for discrete Hamiltonian systems. Comput. Math. Appl. 62(8), 3015-3026 (2011)

2. Tang, XH, Zhang, Q, Zhang, M: Lyapunov-type inequalities for the first-order nonlinear Hamiltonian systems. Comput. Math. Appl. 9, 3603-3613 (2011)

3. He, X, Zhang, Q: A discrete analogue of Lyapunov-type inequalities for nonlinear difference systems. Comput. Math. Appl. 2, 677-684 (2011)

4. Zhang, Q, He, X, Jiang, J: On Lyapunov-type inequalities for nonlinear dynamic systems on time scales. Comput. Math. Appl. 11, 4028-4038 (2011)

5. Ünal, M, Cakmak, D, Tiryaki, A: A discrete analogue of Lyapunov-type inequalities for nonlinear systems. Comput. Math. Appl. 55, 2631-2642 (2008)

6. Zhang, Q, Tang, XH: Lyapunov inequalities and stability for discrete linear Hamiltonian systems. J. Differ. Equ. Appl. 18(9), 1467-1484 (2012)

doi:10.1186/1687-1847-2013-16

Cite this article as: Zhang et al.: Lyapunov-type inequalities and disconjugacy for some nonlinear difference system. Advances in Difference Equations 2013 2013:16.

Submit your manuscript to a SpringerOpen ${ }^{\circ}$ journal and benefit from:

- Convenient online submission

Rigorous peer review

- Immediate publication on acceptance

Open access: articles freely available online

- High visibility within the field

- Retaining the copyright to your article

Submit your next manuscript at $>$ springeropen.com 